\title{
THE NON-EXISTENCE OF A CERTAIN TYPE OF ODD PERFECT NUMBER
}

\author{
R. J. LEVIT
}

For any perfect number ${ }^{1}$ expressed in the form $n=a_{0} a_{1} \cdots a_{t}$, where

$$
a_{0}=p_{0}^{\alpha_{0}}, a_{1}=p_{1}^{\alpha_{1}}, \cdots, a_{t}=p_{t}^{\alpha_{t}}
$$

and $p_{0}, p_{1}, \cdots, p_{t}$ are the distinct prime factors of $n$, it can be shown that a unique one of the prime powers $a_{i}$ has an even divisor sum $\sigma\left(a_{i}\right)$. Throughout we shall suppose that the primes $p_{i}$ and hence the prime powers $a_{i}$ to be so numbered that

$$
\sigma\left(a_{0}\right) \equiv 0 ; \quad \sigma\left(a_{i}\right) \equiv 1 \quad i=1,2, \cdots, t,(\bmod 2) .
$$

Then with the abbreviations

$$
\sigma_{0}=\sigma\left(a_{0}\right) / 2 ; \quad \sigma_{i}=\sigma\left(a_{i}\right), \quad i=1,2, \cdots, t,
$$

the condition for $n$ to be perfect may be written in the form

$$
\sigma(n) / 2=\sigma_{0} \sigma_{1} \cdots \sigma_{t}=a_{0} a_{1} \cdots a_{t}=n .
$$

For the even perfect numbers, which are the only kind known, it is well known that $p_{0}=2^{q}-1, \alpha_{0}=1, p_{1}=2, \alpha_{1}=q-1, t=1$, where $q$ is any prime such that $2^{q}-1$ is also prime. Then $\sigma_{1}=2^{q}-1=a_{0}$ and $\sigma_{0}=2^{q-1}=a_{1}$ so that $\sigma_{0}$ and $\sigma_{1}$ are the prime powers $a_{0}$ and $a_{1}$ in rereverse order. It is natural to inquire whether there may exist odd perfect numbers such that analogously $\sigma_{0}, \sigma_{1}, \ldots, \sigma_{t}$ are the prime powers $a_{0}, a_{1}, \cdots, a_{t}$ in a different order. In the following it will be proved that no odd perfect numbers of this form can exist.

We first establish an algebraic identity. Throughout this paper the product notation $\prod_{i=a}^{b} x_{i}$ is used with the convention that $\prod_{i=a}^{b} x_{i}=1$ if $a>b$.

LEMMA 1. Let $c_{1}, c_{2}, \cdots, c_{t}$ be any $t \geqq 2$ integers (more generally, elements of a commutative ring with a unit element). Then,

Presented to the Society, February 22, 1947; received by the editors September 16, 1946.

1 For a summary of results concerning perfect numbers (including those cited above) with references see L. Dickson, History of the theory of numbers, vol. 1, 1919, pp. 1-33. For a more recent paper with references to other recent literature on the subject, see A. Brauer, On the non-existence of odd perfect numbers of form $p^{\alpha} q_{1}^{2} q_{2}^{2}$. . $q_{t-1}^{2} q_{t}^{4}$, Bull. Amer. Math. Soc. vol. 49 (1943) pp. 712-718. 


$$
\sum_{j=1}^{t}\left[\prod_{i=1}^{j-1}\left(c_{i}-1\right) \prod_{i=j+1}^{t} c_{i}\right]=\prod_{i=1}^{t} c_{i}-\prod_{i=1}^{t}\left(c_{i}-1\right) .
$$

Proof. The identity holds for $t=2$, both members reducing to $c_{1}+c_{2}-1$. Proceeding by induction, assume the identity holds for $t=m$. Multiplying both members by $c_{m+1}$ and adding $\prod_{i=1}^{m}\left(c_{i}-1\right)$, we have

$$
\begin{aligned}
\sum_{j=1}^{m+1}\left[\prod_{i=1}^{j-1}\right. & \left.\left(c_{i}-1\right) \prod_{i=j+1}^{m+1} c_{i}\right] \\
& =c_{m+1} \sum_{j=1}^{m}\left[\prod_{i=1}^{j-1}\left(c_{i}-1\right) \prod_{i=1+1}^{m} c_{i}\right]+\prod_{i=1}^{m}\left(c_{i}-1\right) \\
& =c_{m+1}\left[\prod_{i=1}^{m} c_{i}-\prod_{i=1}^{m}\left(c_{i}-1\right)\right]+\prod_{i=1}^{m}\left(c_{i}-1\right) \\
& =\prod_{i=1}^{m+1} c_{i}-\prod_{i=1}^{m+1}\left(c_{i}-1\right),
\end{aligned}
$$

the first and last members of which are the members of the required identity for $t=m+1$, thus completing the induction.

For an odd integer $n=a_{0} a_{1} \cdots a_{t}$ to be perfect a well known necessary condition is that with the $p$ 's numbered according to (1)

$$
\alpha_{0} \equiv p_{0} \equiv 1(\bmod 4) \text {. }
$$

For such prime powers we have the following:

LEMMA 2. Let $\alpha>1$ be an integer and $p$ a prime such that $\alpha \equiv p \equiv 1$ $(\bmod 4)$. Then $\sigma\left(p^{\alpha}\right)$ is divisible by at least two distinct odd primes.

Proof. It is sufficient to exhibit two odd nontrivial divisors of $\sigma\left(p^{\alpha}\right)$ which are relatively prime. We have

$$
\sigma\left(p^{\alpha}\right)=\frac{p^{\alpha+1}-1}{p-1}=2 \cdot \frac{p^{(\alpha+1) / 2}+1}{2} \cdot \frac{p^{(\alpha+1) / 2}-1}{p-1}
$$

Then the required divisors are

$$
d_{1}=\frac{p^{(\alpha+1) / 2}+1}{2} \text { and } d_{2}=\frac{p^{(\alpha+1) / 2}-1}{p-1} .
$$

They are both odd since $\sigma\left(p^{\alpha}\right)=1+p+p^{2}+\cdots+p^{\alpha} \equiv \alpha+1 \equiv 2$ $(\bmod 4)$. They are coprime, since $2 d_{1}-(p-1) d_{2}=2$ so that if there were a common divisor of $d_{1}$ and $d_{2}$, it would have to divide 2 . Finally, they are nontrivial divisors since $d_{1}>d_{2}>1$ for $\alpha>1$.

We are now able to prove our theorem. 
THEOREM. Let $n=a_{0} a_{1} \cdots a_{t}$, where

$$
a_{0}=p_{0}^{\alpha_{0}}, a_{1}=p_{1}^{\alpha_{1}}, \cdots, a_{t}=p_{t}^{\alpha_{t}}
$$

and $p_{0}, p_{1}, \cdots, p_{t}$ are distinct odd primes. Then, if each of the quantities $\sigma_{i}, i=0,1, \cdots, t$, defined in (2) is a power of a prime, $n$ is not a perfect number.

Proof. Assume that $n$ is perfect. Then (3) holds; and since the $\sigma_{i}$ are prime powers, by the fundamental theorem of arithmetic, they must each equal one of the $a_{j}, i, j=0, \cdots, t$ with $i \neq j$, because $\sigma_{i} \equiv 1 \not \equiv 0\left(\bmod p_{i}\right)$. That is, $\sigma_{0}, \cdots, \sigma_{t}$ are the prime powers $a_{0}, \cdots, a_{t}$ in a different order.

Without loss of generality we may suppose that the $p$ 's are numbered recursively in the following manner: $p_{0}$ has already been chosen in accord with (1) (or (5), which amounts to the same thing). Choose as $p_{1}$ that prime $p_{i}$ for which $a_{i}=\sigma_{0}$, as $p_{2}$ that prime $p_{j}$ for which $a_{j}=\sigma_{1}$, and in general choose as $p_{m}$ that prime $p_{r}$ for which $a_{r}=\sigma_{m-1}$. This process can be continued until a prime $p_{k}$ is reached such that $\sigma_{k}=a_{l}$ with $l<k$ so that we cannot set $a_{k+1}=\sigma_{k}$. We shall now show that this cannot occur until the primes have been completely numbered; that is, when $k=t$, and then $l=0$. First suppose $0<l<k \leqq t$. Then we have both $\sigma_{l}=a_{l}$ and $\sigma_{l-1}=a_{l}$ so that in the product, $\sigma_{0} \cdots \sigma_{l} \cdots \sigma_{k} \cdots \sigma_{t}, p_{l}$ occurs to at least the power $2 \alpha_{l}$ contrary to (3). Next suppose $l=0$ but $k<t$. Then $\sigma_{k}=a_{0}$, and

$$
a_{1} a_{2} \cdots a_{k} a_{0}=\sigma_{0} \sigma_{1} \cdots \sigma_{k-1} \sigma_{k} .
$$

Hence from (3), numbering the $p_{m}, m=k+1, k+2, \cdots, t$ in any order,

$$
a_{k+1} a_{k+2} a_{t}=\sigma_{k+1} \sigma_{k+2} \cdots \sigma_{t} .
$$

But this is impossible, since

$$
\begin{aligned}
\sigma_{k+1} \sigma_{k+2} & \cdots \sigma_{t} \\
= & \left(1+p_{k+1}+p_{k+1}^{2}+\cdots+p_{k+1}^{\alpha_{k+1}}\right)\left(1+\cdots+p_{k+2}^{\alpha_{k+2}}\right) \\
& \quad \cdots\left(1+\cdots p_{t}^{\alpha t}\right)>p_{k+1}^{\alpha_{k+1} p_{k+2}} \cdots p_{k+2}^{\alpha t} \\
= & a_{k+1} a_{k+2} \cdots a_{t} .
\end{aligned}
$$

The only remaining possibility is, then, $k=t, l=0$. Thus the $p$ 's, and hence the $a$ 's, have been completely numbered as follows:

$$
a_{m}=\sigma_{m-1}, \quad m=1,2, \cdots, t ; \quad a_{0}=\sigma_{t} .
$$

In view of (5) and Lemma 2, we must have $\alpha_{0}=1$, since otherwise 
$\sigma_{0}$ would not be a power of a prime.

Now, evaluating the $\sigma$ 's, equations (11) become

$$
\begin{aligned}
a_{1}=\frac{p_{0}+1}{2} ; \quad a_{m} & =\frac{p_{m-1}^{\alpha_{m-1}+1}-1}{p_{m-1}-1}, \quad m=2,3, \cdots, t ; \\
p_{0} & =\frac{p_{t}^{\alpha_{t}+1}-1}{p_{t}-1} .
\end{aligned}
$$

With the definitions,

$$
a_{m}=p_{m}^{\alpha_{m}}, \quad b_{m}=\frac{1}{p_{m}-1}, \quad m=1,2, \cdots, t,
$$

equations (12) become

$$
\begin{gathered}
a_{m}=b_{m-1} p_{m-1} a_{m-1}-b_{m-1} \quad \text { for } m=2,3, \cdots, t \\
a_{1}=\frac{p_{0}+1}{2}, \quad p_{0}=b_{t} p_{t} a_{t}-b_{t} .
\end{gathered}
$$

Eliminating $p_{0}$ from (15) gives

$$
2 a_{1}-1=b_{t} p_{t} a_{t}-b_{t} .
$$

By repeated application of the recursion formula (14) we find that

$$
a_{m}=\left(\prod_{i=1}^{m-1} b_{i} p_{i}\right) a_{1}-\sum_{j=1}^{m-1} b_{j} \prod_{i=j+1}^{m-1} b_{i} p_{i}, \quad m=2,3, \cdots, t,
$$

which is readily verified by induction. From (16) and (17) with $m=t$

$$
2 a_{1}-1=\left(\prod_{i=1}^{t} b_{i} p_{i}\right) a_{1}-\sum_{j=1}^{t} b_{j} \prod_{i=j+1}^{t} b_{i} p_{i}
$$

or

$$
\left(\prod_{i=1}^{t} b_{i} p_{i}-2\right) a_{1}-\sum_{j=1}^{t} b_{j} \prod_{i=j+1}^{t} b_{i} p_{i}+1=0 .
$$

Multiplying by $\prod_{i=1}^{t}\left(p_{i}-1\right)$ and using (13), (19) becomes

$$
\begin{gathered}
{\left[\prod_{i=1}^{t} p_{i}-2 \prod_{i=1}^{t}\left(p_{i}-1\right)\right] a_{1}-\sum_{j=1}^{t}\left[\prod_{i=1}^{j-1}\left(p_{i}-1\right) \prod_{i=j+1}^{t} p_{i}\right]} \\
+\prod_{i=1}^{t}\left(p_{i}-1\right)=0 .
\end{gathered}
$$


Utilizing the identity (4), (20) becomes

$$
\left[\prod_{i=1}^{t} p_{i}-2 \prod_{i=1}^{t}\left(p_{i}-1\right)\right] a_{1}-\left[\prod_{i=1}^{t} p_{i}-2 \prod_{i=1}^{t}\left(p_{i}-1\right)\right]=0
$$

so that

$$
\left(a_{1}-1\right)\left[\prod_{i=1}^{t} p_{i}-2 \prod_{i=1}^{t}\left(p_{i}-1\right)\right]=0 .
$$

Hence, either

$$
p_{1}^{\alpha_{1}}=a_{1}=1
$$

or

$$
\prod_{i=1}^{t} p_{i}=2 \prod_{i=1}^{t}\left(p_{i}-1\right) .
$$

(23) is impossible since $p_{1} \geqq 3$. (24) is also impossible, since the right member is even while the left member, being the product of odd primes, is odd. Thus the assumption that $n$ is perfect leads to a contradiction, and the theorem is proved.

Our results may evidently be restated in the following form:

COROLlaRY. If $n=a_{0} a_{1} \cdots a_{t}$ is an odd perfect number, at least two of the divisor sums $\sigma\left(a_{i}\right)$ must have a common factor greater than 1 .

UNIVERSITY OF GEORgIA 